D) Check for updates

Cite this: Nanoscale, 2021, 13, 9904

DOI: $10.1039 / \mathrm{d} 1 \mathrm{nr} 90103 f$

rsc.li/nanoscale

\title{
Advanced nanomaterials for energy conversion and storage: current status and future opportunities
}

\author{
Wee-Jun Ong, (D) *a,b,c Nanfeng Zheng ${ }^{* d}$ and Markus Antonietti*e
}

Energy science has witnessed a surge of interest over the past 10 years, mostly motivated by progress in nanoscience and nanotechnology. For the sustainable development of human beings, extensive research has been dedicated to renewable energy, and its conversion and storage, owing to the increasing concerns about global climate change and the growing demand for energy. In April 2021, the $\mathrm{CO}_{2}$ concentration was measured at $418.46 \mathrm{ppm}$, and compared to April 2020 (415.59 ppm) even through coronavirus times the increase is massive. As a result, searching for promising new options is crucial to align human development with the United Nation's Sustainable Development Goals.

In particular, downsizing functional materials to the nanoscale can manifest intriguing properties and performances compared to their bulk structures.

\footnotetext{
${ }^{a}$ School of Energy and Chemical Engineering, Xiamen University Malaysia, Selangor Darul Ehsan 43900, Malaysia.E-mail:weejun.ong@xmu.edu.my ${ }^{b}$ Center of Excellence for NaNo Energy \& Catalysis Technology (CONNECT), Xiamen University Malaysia, Selangor Darul Ehsan 43900, Malaysia ${ }^{c}$ College of Chemistry and Chemical Engineering, Xiamen University, Xiamen 361005, China ${ }^{d}$ State Key Laboratory for Physical Chemistry of Solid Surfaces, Collaborative Innovation Center of Chemistry for Energy Materials, and National \& Local Joint Engineering Research Center for Preparation Technology of Nanomaterials, College of Chemistry and Chemical Engineering, Xiamen University, Xiamen 361005, China.E-mail: nfzheng@xmu.edu.cn ${ }^{e}$ Max-Planck Institute of Colloids and Interfaces, Department of Colloid Chemistry, Research Campus Golm, Am Mühlenberg 1, 14476 Potsdam, Germany. E-mail: markus.antonietti@mpikg.mpg.de
}

Fabricating nanostructured materials with tailored properties is at the forefront of technological exploration. ${ }^{1}$ At present, novel strategies such as size/ facet control, structural engineering, vacancy engineering, atomic regulation, and construction of nanocomposites alter the physicochemical properties (e.g. electronic, optical, band and textural) of the active sites. ${ }^{2,3}$ Hence, this gives rise to a momentous improvement in the performance of nanomaterials toward energy conversion and storage. Research in this energy realm necessitates an interdisciplinary approach with synergistic collaboration from all disciplines such as chemistry, engineering, nanotechnology, computation, as well as industrial thinking to accomplish highperformance energy systems.

The themed collection of Nanoscale entitled "advanced nanomaterials for energy conversion and storage" aims to showcase the state-of-the-art knowledge on the development of nanomaterials with tunable properties for diverse energy applications. This themed collection consists of 23 Full Papers, 4 Communications and 5 Reviews, focusing on designing advanced materials and building a structure-activity-stability relationship in electrocatalysis, photocatalysis, photoelectrocatalysis, batteries, fuel cells and so forth.

Xiong et al. (DOI: 10.1039/ D0NR02596H) highlight the development of engineering active sites on surfaces and in open frameworks with respect to surface vacancies, doped heteroatoms, loaded metal nano- particles, crystal facets and metal nodes/ organic linkers in metal-organic frameworks for application in photocatalytic $\mathrm{CO}_{2}$ reduction. In addition to the advances in $\mathrm{CO}_{2}$ photoreduction, Zhang et al. (DOI: 10.1039/D0NR03178J) have reviewed the use of carbon-based nanomaterials and their hybrids for photoand electrocatalytic hydrogen peroxide $\left(\mathrm{H}_{2} \mathrm{O}_{2}\right)$ production via both reductive and oxidative reaction pathways. Apart from photochemistry, inspired by the merits of 2D nanostructures, Tsang's group (DOI: 10.1039/D0NR01295E) present a minireview on the recent discoveries in hetero-single atom-doped $\mathrm{MoS}_{2}$ nanosheets for electrochemical hydrogen evolution reaction (HER) from water by reviewing the nature of the dopants, doping positions and the polytypes of $\mathrm{MoS}_{2}$. In view of the importance of morphological engineering in energy applications, Wang et al. (DOI: 10.1039/ D0NR03425H) focus on the primary issues facing one-dimensional (1D) electrospun carbon nanofibers in supercapacitors with the aim of ameliorating the conductivity, modulating pore configuration, doping with heteroatoms and increasing mechanical strength. Sun et al. (DOI: 10.1039/D0NR05475E) summarize the most recent updates on the structure-activity relationship of random alloy and intermetallic (ordered structure) nanocrystals for electrochemical fuel cells with robust activity and superb stability.

By mimicking natural photosynthesis, artificial photosynthesis using nanocatalysts is described by several research groups. ${ }^{4}$ For enhancing the light absorp- 
tion and inhibiting the electron-hole recombination, morphological modification and surface engineering are facile techniques to boost photocatalysis. ${ }^{5-7}$ Tang et al. (DOI: 10.1039/D0NR00226G) have prepared graphitic carbon embedded inside hollow graphitic carbon nitride $\left(\mathrm{g}-\mathrm{C}_{3} \mathrm{~N}_{4}\right)$. Yu's group (DOI: 10.1039/C9NR10451H) successfully developed hierarchical $\mathrm{Ni}-\mathrm{NiS} / \mathrm{C} / \mathrm{ZnO}$ photocatalysts via in situ photodeposition of Ni-NiS nanosheets onto $\mathrm{C} / \mathrm{ZnO}$ electrospun nanofibers for $\mathrm{CO}_{2}$ reduction to $\mathrm{CO}$ and $\mathrm{CH}_{4}$. Attributed to the advantages of $2 \mathrm{D} / 2 \mathrm{D}$ heterojunction systems, Jing et al. (DOI: 10.1039/ D0NR02551H) fabricated dimensionmatched ultrathin NiMOF/g- $\mathrm{C}_{3} \mathrm{~N}_{4}$ heterojunctions with the aid of ultrasound by

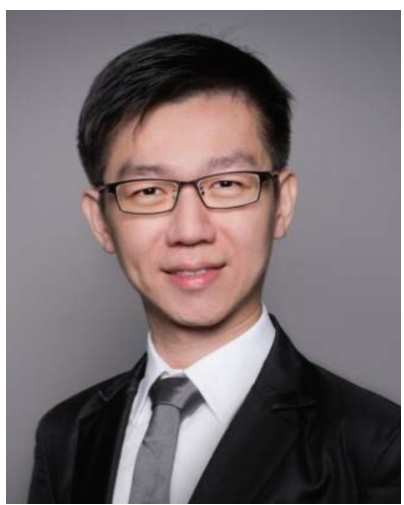

Wee-Jun Ong

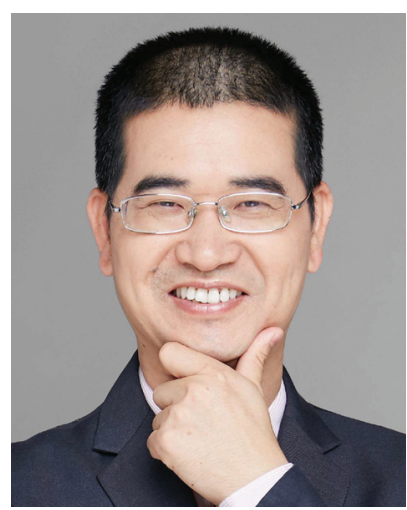

Nanfeng Zheng growing NiMOF nanosheets on hydroxylated and 1,4-aminobenzoic acid-functionalized $\mathrm{g}-\mathrm{C}_{3} \mathrm{~N}_{4}$ nanosheets for improved $\mathrm{CO}_{2}$ reduction. To aim for energy sustainability as opposed to the energyintensive industrial Haber-Bosch process, Tang et al. (DOI: 10.1039/ D0NR02527E) designed a ternary heterostructure consisting of ruthenium species on $\mathrm{g}-\mathrm{C}_{3} \mathrm{~N}_{4}\left(\mathrm{Ru} / \mathrm{RuO}_{2} / \mathrm{g}-\mathrm{C}_{3} \mathrm{~N}_{4}\right)$ for ammonia photosynthesis, in which $\mathrm{Ru}$ and $\mathrm{RuO}_{2}$ functioned as electron and hole storage sites, respectively. Furthermore, Zhang et al. (DOI: 10.1039/ D0NR03393F) report W-doped $\mathrm{TiO}_{2}$ for boosted photothermocatalytic $\mathrm{CO}_{2}$ reduction to $\mathrm{CO}$ due to the presence of more active sites with increased $\mathrm{W}$ doping. By applying an external bias,
Jorge et al. (DOI: 10.1039/D0NR06139E) introduce a carbon underlayer derived from carbon dots via a hydrothermal process between the fluorine-doped tin oxide substrate and the hematite photoanodes, which has remarkably enhanced the photocurrent density and charge transfer efficiency of up to $c a$. $80 \%$ at 1.25 $\mathrm{V}$ vs. RHE. Besides energy conversion, An's group (DOI: 10.1039/D0NR01027H) has synthesized well-aligned 2D Ni-MOF nanosheet arrays vertically grown on porous nickel foam (Ni-MOF/NF) without lateral stacking via solvothermal processes for the removal of ethyl acetate. Falaras's group (DOI: 10.1039/ D0NR02562C) designed an innovative interface engineering approach to utilize an organic chromophore as an
Wee-Jun Ong received his B.Eng. and Ph.D. in chemical engineering from Monash University. He is an Associate Professor in School of Energy and Chemical Engineering at Xiamen University Malaysia. Starting from 2021, he has become a Director of Center of Excellence for NaNo Energy \& Catalysis Technology (CONNECT). Previously, he was a scientist at Agency for Science, Technology and Research ( $\left.A^{*} S T A R\right)$, Singapore. In 2019, he was a visiting scientist at Technische Universität Dresden, Germany and a visiting professor at Lawrence Berkeley National Laboratory (LBNL), USA. His research interests include surface/atomic engineering of nanomaterials for energy storage devices, photocatalytic, photoelectrocatalytic, and electrochemical $\mathrm{H}_{2} \mathrm{O}$ splitting, $\mathrm{CO}_{2}$ reduction and $\mathrm{N}_{2}$ fixation.
Nanfeng Zheng received his B.S. from Xiamen University in 1998. In 2005, he obtained his Ph.D. degree from University of California - Riverside. During 2005-2007, he worked as a research associate at University of California Santa Barbara. He moved to Xiamen University as a Full Professor in 2007 and became a Changjiang Chair Professor in 2010. He is currently the executive deputy director of Innovation Laboratory for Sciences and Technologies of Energy Materials of Fujian Province (IKKEM). His research is committed to understanding the surface and interface chemistry behind the factors regulating the properties of functional materials at the molecular level and thus promoting their applications in the fields of energy, catalysis, and environmental protection.

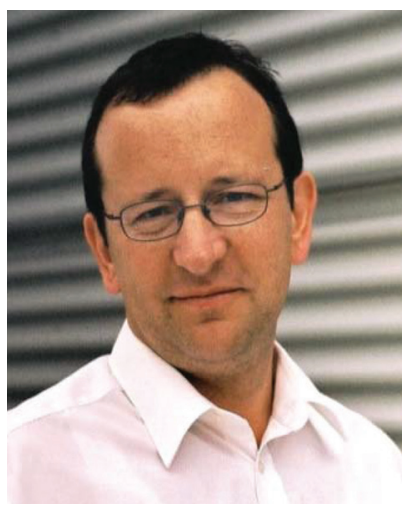

Markus Antonietti

Markus Antonietti is the Materials Chemistry Director of the Max Planck Institute of Colloids and Interfaces in Potsdam-Golm, Germany. He is an expert in polymers and covalent materials and has focused his attention in the last years to the fields of green chemistry and sustainable materials, but also to the new global cycles of energy, food, water, and $\mathrm{CO}_{2}$ constituting the Anthropocene. In his free time, he enjoys cooking and performing in a rock band. 
interlayer between a perovskite absorber and hole transporter, which preserved $83 \%$ of the original efficiency despite storing the device for 37 days in the dark and under open-circuit conditions.

In addition to light-driven reactions, research in the field of electrocatalysis for water splitting and $\mathrm{CO}_{2}$ reduction is a prime focus of sustainable energy research. ${ }^{8-11}$ As a low-cost alternative to Pt, Yamashita's group (DOI: 10.1039/ D0NR02525A) employed the noblemetal-free hybrid phase $1 \mathrm{~T} / 2 \mathrm{H}-\mathrm{MoS}_{2}$ with tunable $1 \mathrm{~T}$ concentration for electrochemical hydrogen evolution. Oh et al. (DOI: 10.1039/D0NR02951C) studied the $\mathrm{pH}$ influence on the electroactivation of IrNi alloy nanoparticles supported on carbon ( $\mathrm{IrNi} / \mathrm{C}$ ) toward water oxidation, where different $\mathrm{pH}$ conditions led to extraordinary electronic structure by modifying the alloy catalysts. Liu et al. (DOI: 10.1039/ D0NR03378B) developed trifunctional electrocatalysts for hydrogen evolution (HER), oxygen evolution (OER) and oxygen reduction reactions (ORR), which are composed of a hierarchically-structured $\mathrm{Pt} / \mathrm{NiO} / \mathrm{Ni} / \mathrm{CNT}$ with around $2 \mathrm{~nm}$ of Pt nanoparticles via substrateenhanced electroless deposition. Beyond water splitting for hydrogen and oxygen evolution reactions, Luo et al. (DOI: 10.1039/D0NR02591G) synthesized nanostructured $\mathrm{Cu} @ \mathrm{Cu}_{2}(\mathrm{OH})_{3} \mathrm{NO}_{3}$ electrodes via a molten salt decomposition method (MSDM) for $\mathrm{CO}_{2}$ electroreduction to $\mathrm{C}_{2} \mathrm{H}_{4}$ in $\mathrm{KHCO}_{3}$ solution with a high faradaic efficiency of $31.8 \%$ and robust stability of over $20 \mathrm{~h}$. In another investigation, Chen's group (DOI: 10.1039/D0NR03475D) unravelled the effect of the dynamic chemical state on the selective $\mathrm{CO}_{2}$ reduction to $\mathrm{CO}$ and formate using $\mathrm{Zn}$ electrocatalysts via in situ Raman spectroscopy, X-ray absorption spectroscopy (XAS) and X-ray diffraction, in which $\mathrm{Zn}(\mathrm{II})$ and $\mathrm{Zn}(0)$ species played a dominant role in CO and formate production, respectively. $\mathrm{Li}$ et al. (DOI: 10.1039/C9NR10304J) employed a series of an electrochemical method and in situ SERS to elucidate the size effects of Pt on the activity of Pt-on$\mathrm{Au}$ nanocatalysts with a Au-core Pt-satellite superstructure toward CO and metha- nol electrooxidation. Other than that, by taking the merits of the appealing optoelectronic and electrochemical attributes of MXene, Gogotsi et al. (DOI: 10.1039/ D0NR02673E) fabricated solution processable transparent conducting electrodes for three-electrode electrochromic cells by employing titanium carbide MXenes. Along another energy conversion route, Hong et al. (DOI: 10.1039/D0NR03303K) examined thermoelectricity in molecular junctions developed from oligophenylene-ethynylene derivatives.

Advances in energy storage devices using nanotechnology is another global trend of energy research. ${ }^{9,12,13} \mathrm{Xu}$ et al. (DOI: 10.1039/D0NR02016H) prepared multilayered nickel-cobalt organic framework (NiCo-MOF) nanosheets as robust electrode materials for excellent electrochemical energy storage over 3000 cycles at $5 \mathrm{~A} \mathrm{~g}^{-1}$. Kovalenko et al. (DOI: $10.1039 /$ D0NR02930K) present the synthesis of homogeneously embedded $\mathrm{Sb}$ nanoparticles in a silicon oxycarbide (SiOC) matrix, which was able to provide a reasonably high Li-ion storage capacity. Hwang et al. (DOI: 10.1039/ D0NR02569K) designed the intercalative hybridization of $\mathrm{MoS}_{2}$ with chromium hydroxide nanoclusters, which increase the basal spacing, accelerate charge kinetics and stabilize the open porous stacking structure for boosted charge storage capacity and rate performance for Li-ion batteries. ${ }^{4}$ In $\mathrm{Li}-\mathrm{S}$ batteries, sluggish dynamics of lithium polysulfides (LiPS) conversion results in fast capacity decay and ineffective utilization of active sulfur. Wu et al. (DOI: 10.1039/ D0NR03528A) designed a hierarchical MXene@TiO ${ }_{2}$ nanoarray via in situ solvothermal strategies, which is able to retain a stable discharge capacity of $612.7 \mathrm{~mA} \mathrm{~h} \mathrm{~g}^{-1}$ after 500 cycles at a rate of $2 \mathrm{C}$ in a Li-S battery. In another related work, a ZnS quantum dot@graphene nanosheet ( $\mathrm{ZnS}$ QD@rGO) catalyst was fabricated by Wei and coworkers (DOI: 10.1039/D0NR02429E) to ameliorate polysulfide conversion for high energy density Li-S batteries. By blocking the LiPS shuttling, the construction of an interlayer with low $\mathrm{Li}$ ion diffusion resistance renders a magnificent approach. Lv et al. (DOI: 10.1039/
D0NR02607G) constructed a thin porous carbon nanosheet with embedded $\mathrm{TiO}_{2}$ nanoparticles as an interlayer on the separator, which allows rapid $\mathrm{Li}$ ion diffusion and simultaneously blocks the polysulfide diffusion. In another work, Lee et al. (DOI: 10.1039/D0NR02258F) engineered a Li metal anode by employing microporous and mesoporous carbon as host materials to avoid degradation during cycling of $\mathrm{Li}-\mathrm{S}$ batteries.

Other than Li batteries, Dong and Wang et al. (DOI: 10.1039/D0NR02604B) reported the design of a walnut-like $\mathrm{MoS}_{2} @ S n s$ core-shell heterostructure as an anode for sodium-ion batteries, which exemplified superior electrochemical performances ascribed to improved ion diffusion at the heterointerface driven by an internal electric field. To circumvent shortcomings present in organic potassium-ion batteries (PIB), Zhang et al. (DOI: 10.1039/D0NR00964D) designed a non-redox-metal potassium metalorganic framework (K-MOF) as an auspicious organic anode for high electrochemical performance and cycling for more than 300 cycles with capacity retention of $92 \%$ stemming from the $\mathrm{N}-\mathrm{K} / \mathrm{O}-$ $\mathrm{K}$ coordination bonds. Apart from the organic anode, Mai's group (DOI: 10.1039/D0NR01274B) constructed selfadaptive $\mathrm{NiS}_{2}$ nanoparticles embedded in three-dimensional (3D) graphene oxide as a robust anode with outstanding rate behaviour and high reversible capacity in PIB with reversible transformation from $\mathrm{NiS}_{2}$ to $\mathrm{K}_{x} \mathrm{NiS}_{2}$ followed by generating $\mathrm{Ni}$ and $\mathrm{K}_{2} \mathrm{~S}_{4}$ products. Bifunctional OER/ ORR electrocatalysts composed of $\mathrm{N}$-doped carbon nanotubes containing NiFe alloy nanoparticles were fabricated by Zhang et al. (DOI: 10.1039/ D0NR02486D), in which an excellent performance for rechargeable zinc-air batteries was ascribed to the high porosity and boosted conductivity, hence benefitting mass and electron transfer processes.

We are certain that this themed collection will inspire the readers to further advance fundamental understanding of the state-of-the-art energy applications to potentially meet the industrial requirements for commercialization and globalization. As Guest Editors, we would like to thank all invited authors for their 
excellent contributions as well as all Nanoscale's editors and reviewers for their selfless professional services. On a final note, in conjunction with the Xiamen University's anniversary in 2021, we would like to commemorate and congratulate on the $100^{\text {th }}$ anniversary of Xiamen University, China and the $5^{\text {th }}$ anniversary of Xiamen University Malaysia campus. In view of the university's anniversary, we would like to celebrate the successful launch of the Center of Excellence for NaNo Energy \& Catalysis Technology (CONNECT) at Xiamen University Malaysia in 2021.

\section{Acknowledgements}

W.-J. Ong acknowledges financial assistance from the Ministry of Higher Education (MOHE) Malaysia under the Fundamental Research Grant Scheme (FRGS) (ref. no: FRGS/1/2020/
TK0/XMU/02/1), Xiamen University Malaysia Investigatorship Grant (grant no: IENG/0038) and Xiamen University Malaysia Research Fund (XMUMRF/ 2019-C3/IENG/0013).

\section{References}

1 R. Qin, K. Liu, Q. Wu and N. Zheng, Chem. Rev., 2020, 120, 11810-11899.

2 W.-J. Ong, L.-L. Tan, Y. H. Ng, S.-T. Yong and S.-P. Chai, Chem. Rev., 2016, 116, 7159-7329.

3 N. Lopez-Salas, J. Kossmann and M. Antonietti, Acc. Mater. Res., 2020, 1, 117-122.

4 C. Dai and B. Liu, Energy Environ. Sci., 2020, 13, 24-52.

5 F. O. Ochedi, D. Liu, J. Yu, A. Hussain and Y. Liu, Environ. Chem. Lett., 2021, 19, 941-967.

6 K. Li, S. Zhang, Y. Li, J. Fan and K. Lv, Chin. J. Catal., 2021, 42, 3-14.
7 X. Yu, S.-F. Ng, L. K. Putri, L.-L. Tan, A. R. Mohamed and W.-J. Ong, Small, 2021, 2006851, DOI: 10.1002/ smll.202006851.

8 Z. Tian, N. López-Salas, C. Liu, T. Liu and M. Antonietti, Adv. Sci., 2020, 7, 2001767.

9 Y. Wang, J. Liu and G. Zheng, Adv. Mater., 2021, 2005798, DOI: 10.1002/ adma.202005798.

10 J.-F. Sun, J.-T. Wu, Q.-Q. Xu, D. Zhou and J.-Z. Yin, Environ. Chem. Lett., 2020, 18, 1593-1623.

11 X. Zhang, A. Chen, L. Chen and Z. Zhou, Adv. Energy Mater., 2021, 2003841, DOI: 10.1002/ aenm.202003841.

12 S.-F. Ng, M. Y. L. Lau and W.-J. Ong, Adv. Mater., 2021, 2008654, DOI: 10.1002/adma.202008654.

13 C. Wu, W.-H. Lai, X. Cai, S.-L. Chou, H.-K. Liu, Y.-X. Wang and S.-X. Dou, Small, 2021, 2006504, DOI: 10.1002/ smll.202006504. 